\title{
Examination of Experimental Method to Extract Characteristics of Lower Limb Movement in Emergency Braking Operation
}

\author{
Masashi Makita1, Kiyoshi Shiroishi' ${ }^{2}$, Hiroaki Fujii ${ }^{3}$ \\ ${ }^{1}$ Faculty of Science and Engineering, Teikyo University, Tochigi, Japan \\ ${ }^{2}$ Faculty of Health Care Medicine, Japan Sports University, Tokyo, Japan \\ ${ }^{3}$ Faculty of Medical Technology, Teikyo University, Tochigi, Japan \\ Email:m.makita@mps.teikyo-u.ac.jp
}

How to cite this paper: Makita, M., Shiroishi, K. and Fujii, H. (2020) Examination of Experimental Method to Extract Characteristics of Lower Limb Movement in Emergency Braking Operation. Engineering, 12, 457-465.

https://doi.org/10.4236/eng.2020.127032

Received: March 16, 2020

Accepted: July 14, 2020

Published: July 17, 2020

Copyright $(92020$ by author(s) and Scientific Research Publishing Inc. This work is licensed under the Creative Commons Attribution International License (CC BY 4.0).

http://creativecommons.org/licenses/by/4.0/

\begin{abstract}
The purpose of this study is to clarify the driving behavior and reaction pattern peculiar to the elderly. In this paper, we conducted a brake operation experiment with five young people, and aimed to create an evaluation index and an experimental method that can extract the characteristics of emergency brake operation from pedaling force and lower limb movement. The results showed that in order to operate the brakes strongly and quickly, the knee was firmly flection, and then the pedaling force was increased when the knee was extended. Furthermore, it was shown that if the brakes were operated without moving the ankle joint, the operation would be quicker.
\end{abstract}

\section{Keywords}

Human Engineering, Older Person, Driver Characteristics, Experiment, Experimental Index

\section{Introduction}

At present, Japan's total population is declining, but the aging rate is rising, and the percentage of the population aged 65 and over in the total population (aging rate) was $27.7 \%$ as of October 2017 . The trend is expected to continue in the future, with the aging rate reaching $38.4 \%$ in 2065 , and one in 2.6 people is predicted to be 65 or older [1]. Social issues associated with this aging include increasing social security benefits such as pensions, medical care, and welfare [2], building a comprehensive regional system to support the elderly, securing human resources necessary for nursing care [3], Although there are various types, 
traffic accidents involving the elderly are also important issues [4].

The number of traffic accidents has decreased for 13 consecutive years since 2005 and the number of fatalities has been decreasing for 6 consecutive years since 2012. On the other hand, in traffic accidents involving the elderly, both the number of incidents and the number of deaths are increasing [5]. The main factors are demographic changes (increase in the elderly population due to the baby boomers reaching 65 years of age or older) [4] [5], and changes in physique due to aging (obesity) [6]) And bone mineral density [7], and decreased driving ability (physical ability) [3]. In this study, this study focuses on the physical ability of the elderly.

In recent years, traffic accidents due to erroneous operation of the brake pedal and accelerator pedal of the elderly, which has become a social problem, have increased [8]. One of the reasons is thought to be the decrease in physical ability due to aging [3] [5].

Research on the arrangement of brake pedals and step-changing operation of automobiles has been conducted for a long time. In examining the arrangement of brake pedals, a study focused on step-changing time [9] and quantification of muscle action during operation by EMG measurement Some studies [10], as well as studies that clarified the characteristics of the difference in the changeover time between young people, the elderly, and men and women [11]. In addition, recently, accident analysis from accidental operation of the brake pedal by elderly people has revealed factors that clarify factors in sitting posture and driving operation [12] [13]. Such research has been focused on the brakes of the elderly and young people, such as deciding the position of the brake pedal, which is less erroneous operation and operation load in designing the vehicle, and enlightening the elderly in safe driving. This is a useful result to reduce traffic accidents due to pedal misoperation. However, there has been a study to quantitatively clarify this by focusing on human physical characteristics such as how to use the muscles and movements of the driver who operates the brake pedal, cognitive functions, and the decline in physical functions with age absent.

Therefore, the purpose of the research is to clarify the effect of deterioration of physical characteristics due to aging on the brake pedal operation (below brake operation) of a car. The relationship between the treading force and the lower limb movement (changes in the angle of the knee and ankle joints) due to the brake operation was examined. In addition, for the actual experiment, we also extracted defects such as measurement items.

Note that the "emergency" brake operation performed in this preliminary experiment does not simulate a specific driving scene of actual driving, but considers the pedaling force exerted in the braking operation to be the force exerted by the lower limbs (muscle strength), and The action was to extract the characteristics of lower limb movements when they occurred. The contents are introduced below. 


\section{Method}

The participants were five healthy men (aged 22 to 55) who had no abnormal motor function. This study was reviewed by the Ethics Committee of Teikyo University, and informed consent was given to experimental participants.

Figure 1 shows a schematic diagram of the experimental apparatus. A real vehicle (without a license plate) that can travel was used for the experiment. And the brake pedal was used as it was. The pedal effort during the brake operation was obtained from the hydraulic pressure of the brake unit, and the lower limb movement was analyzed from the side of the driver's seat with a high-speed camera (250 fps) to capture the movement of the lower limb during braking. After that, the images taken with FrameDIAZV (manufactured by DKH) were digitized to obtain two-dimensional coordinate values, then converted to real length, and the knee and ankle angles were calculated. In addition, a lamp (LED type synchronizer @ DKH) was installed so as to be positioned in front of the driver's seat as a signal to start the brake operation simulating "urgent".

Participants were seated on the test vehicle seats, and the position of the seats was adjusted as much as possible to make it as easy to drive as possible. Next, the trainer was given approximately 15 minutes of practice time to get used to operating the operating devices (brake, accelerator pedal, steering, etc.) of the experimental vehicle.

In the experiment, the braking operation was performed by static operation, which operates slowly without regard to speed, and dynamic operation, which simulated "emergency". The static operation was a trial in which the maximum stepping force at which the muscular strength of the lower limbs was generated was performed, and the player stepped all the way without worrying about the speed once. Next, in the dynamic operation simulating "urgent", when the lamp (in Figure 1) was lit, the operation of depressing twice strongly as soon as possible was performed twice. At that time, the acquisition of treading force data was performed as static and dynamic operations, and the lower limb movement was photographed only with dynamic operations.

\section{Results}

Figure 2 shows the time history chart of the pedaling force. The maximum value

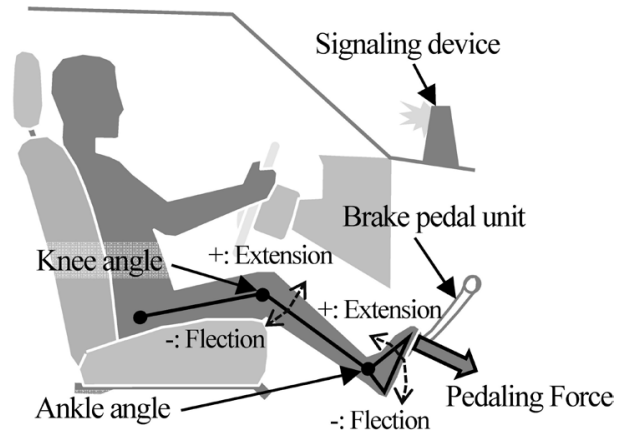

Figure 1. Setup of car experiment and measurement value. 
was read for the static treading force, and the maximum value was read for the other dynamic treading force as the treading force (the maximum value of the initial gradient) instantaneously generated by the participants. The joint angles of the lower limbs (knee and ankle joints) were defined as the maximum amount of change in the bending and extension of each joint during brake operation, based on the joint angle before operation.

First, as shown in Figure 3, features were extracted from the relationship between the ratio [\%] of the dynamic maximum pedaling force to the static and the change in the angle of the lower limb (knee/foot joint). Table 1 shows the correlation coefficient between the leg force [\%] and the lower limb angle (Knee and Ankle flection and extension). In this paper, we used Pearson's product-moment correlation coefficient to determine the relationship between the two variables, and determined that a risk factor (P-value) of less than $5 \%$ was significant.

As a result, there was a strong correlation between the ratio of treading force and knee flexion $(\mathrm{r}=0.843, \mathrm{p}<0.05)$ and the ratio of treading force and knee flexion + extension $(r=0.719, \mathrm{p}<0.05)$. Although there was no statistical significance between the ratio of the treading force and the flexion + extension of the ankle joint, there was a tendency that the greater the ratio of the treading force,

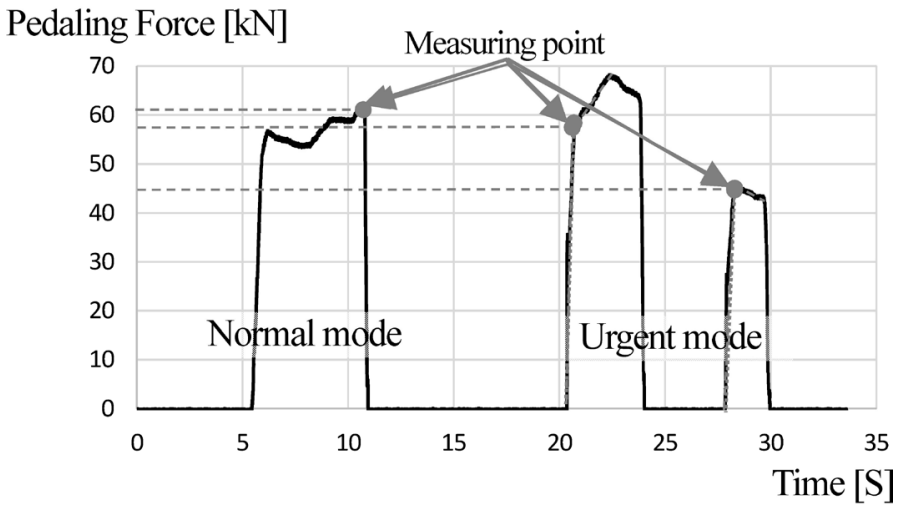

Figure 2. Measuring point of pedaling force-time history.

\section{Lower limb movement [degree]}

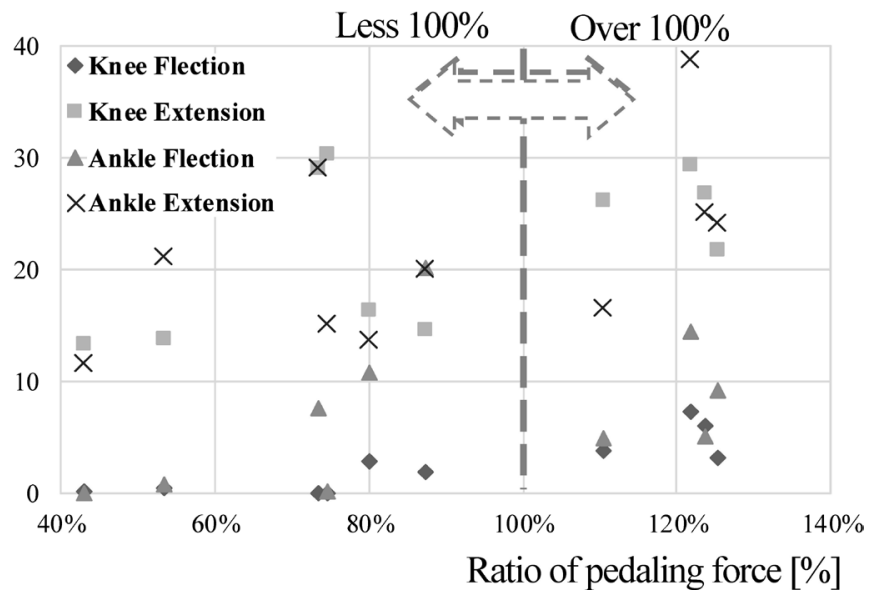

Figure 3. Scatter plot of Ratio of pedaling force and Lower limb movement. 
the greater the ankle plantar flexion movement.

Furthermore, when the relationship between the lower limb joint angles for Less $100 \%$ (Dynamic is lower than static) and Over 100\% (Dynamic is higher than static) was calculated, there was no statistically significant correlation between them. However, participants who were Less $100 \%$ tended to increase their ankle flexion.

Next, the instantaneous force $[\mathrm{kgf} / \mathrm{s}]$ was defined as the slope obtained by dividing the dynamic pedaling force of $10 \mathrm{kgf}$ by the time of occurrence. Figure 4 shows the relationship between the instantaneous force [kgf/s] and the lower limb movement (Knee and Ankle flection and extension).

Table 2 shows the correlation coefficient between the instantaneous power [kgf $/ \mathrm{s}]$ and the lower limb movement angle (Knee and Ankle flection and extension).

As a result, a strong negative correlation was found between the instantaneous power and the ankle flexion $(\mathrm{r}=-0.670, \mathrm{p}<0.05)$, and between the instantaneous power and the ankle movement (flection and extension $r=0.748, p<0.05$ ), there was a moderate correlation between the instantaneous force and extension

Table 1. Relationship between lower limb joint kinematics and pedaling force during braking movement.

\begin{tabular}{cccccccc}
\hline & \multicolumn{2}{c}{ Knee } & \multicolumn{2}{c}{ Ankle } & \multicolumn{2}{c}{ Flection + Extension } \\
\cline { 2 - 7 } & $\begin{array}{c}\text { Knee } \\
\text { Flection }\end{array}$ & $\begin{array}{c}\text { Knee } \\
\text { Extension }\end{array}$ & $\begin{array}{c}\text { Ankle } \\
\text { lection }\end{array}$ & $\begin{array}{c}\text { Ankle } \\
\text { Extension }\end{array}$ & Knee & Ankle \\
\hline Correlation coefficient & 0.843 & 0.54 & 0.445 & 0.546 & 0.719 & 0.59 \\
P-value & 0.011 & 0.110 & 0.206 & 0.105 & 0.017 & 0.073 \\
Less 100\% Correlation coefficient & 0.559 & 0.346 & 0.773 & 0.264 & 0.459 & 0.690 \\
P-value & 0.274 & 0.531 & 0.075 & 0.639 & 0.390 & 0.142 \\
Over 100\% Correlation coefficient & 0.242 & -0.249 & 0.385 & 0.530 & -0.066 & 0.494 \\
P-value & 0.805 & 0.799 & 0.684 & 0.555 & 0.947 & 0.588 \\
\hline
\end{tabular}

Lower limb movement [degree]

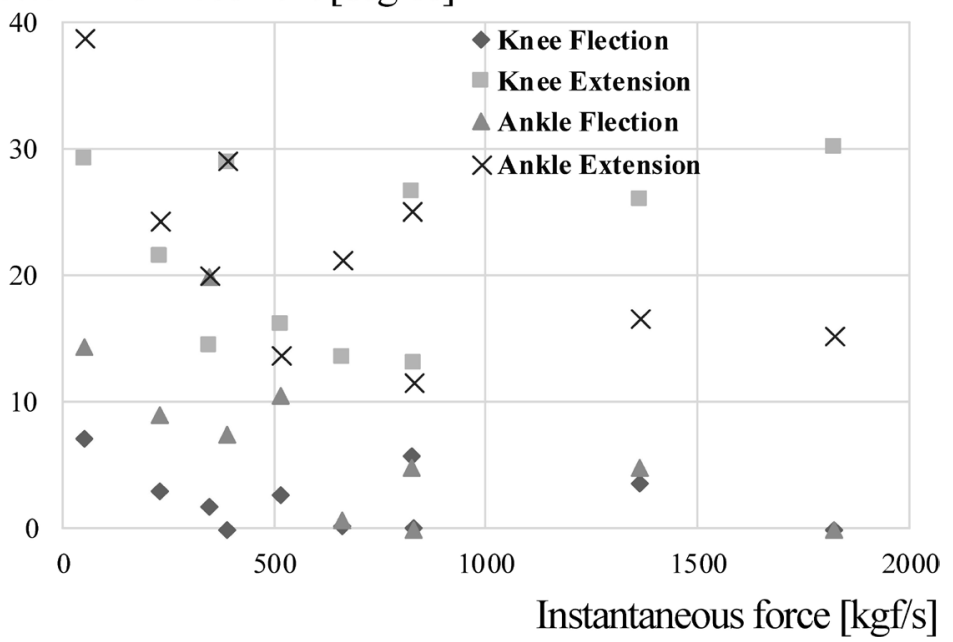

Figure 4. Scatter plot of Instantaneous force and Lower limb movement. 
Table 2. Relationship between lower limb joint kinematics and instantaneous pedaling force during braking.

\begin{tabular}{cccccccc}
\hline & \multicolumn{2}{c}{ Knee } & \multicolumn{2}{c}{ Ankle } & \multicolumn{2}{c}{ Flection + Extension } \\
\cline { 2 - 8 } & $\begin{array}{c}\text { Knee } \\
\text { Flection }\end{array}$ & $\begin{array}{c}\text { Knee } \\
\text { Extension }\end{array}$ & $\begin{array}{c}\text { Ankle } \\
\text { Flection }\end{array}$ & $\begin{array}{c}\text { Ankle } \\
\text { Extension }\end{array}$ & Knee & Ankle \\
\hline Correlation coefficient & -0.340 & 0.255 & -0.670 & -0.605 & 0.110 & 0.748 \\
P-value & 0.350 & 0.491 & 0.032 & 0.063 & 0.770 & 0.011 \\
Less 100\% Correlation coefficient & -0.455 & 0.500 & -0.627 & -0.439 & 0.458 & 0.685 \\
P-value & 0.395 & 0.342 & 0.202 & 0.415 & 0.391 & 0.146 \\
Over 100\% Correlation coefficient & -0.385 & 0.004 & -0.867 & -0.831 & 0.147 & 0.863 \\
P-value & 0.685 & 0.997 & 0.186 & 0.234 & 0.882 & 0.192 \\
\hline
\end{tabular}

of the ankle $(\mathrm{r}=-0.605, \mathrm{p}=0.063)$. Furthermore, there was no correlation between lower limb movements (Knee and Ankle) for Less 100\% and Over 100\%.

\section{Discussion}

Static and dynamic brake operation experiments were conducted by five participants, and the characteristics of the change in the angle of the lower limbs (knee and ankle joint) with respect to the stepping force ratio [\%] and the instantaneous force [kgf/s] were extracted. The characteristic of the change in the angle of the lower limb (knee and ankle joint) with respect to the percentage of the treading force [\%] is that there is a strong correlation between knee flexion and flexion + extension. It is considered that you are operating.

In addition, we tried to extract the characteristics of the pedaling force ratio [\%] for each of Less $100 \%$ and Over $100 \%$. However, no useful results were obtained. One of the reasons is that this paper is a preliminary experiment and the number of participants is small. In the future, it is necessary to increase the number of participants in the experiment through additional tests and to improve the accuracy of feature extraction from the acquired data.

Next, regarding the characteristics of the change in the angle of the lower limb (knee/ankle) with respect to the instantaneous force $[\mathrm{kgf} / \mathrm{s}]$, the higher the instantaneous force, the smaller the flexion movement of the ankle joint. It became clear that the bending motion was small. This suggests that the brake was applied so that the lower limbs were pushed forward with the hips without bending the ankle joint and then straightening the leg without extending the ankle joint. The video analysis was attempted on the result of suspicion of the brake operation (braking operation by stretching the lower limb or body between the seat and the brake pedal) due to the movement of the trunk and thigh (extension of the hip joint) was difficult. Therefore, in future follow-up tests, it is necessary to improve the extraction accuracy of features from the acquired data by adding measurement items such as the angle change of the hip joint and the relative position between the experiment participant and the seat. 
Furthermore, the relationship between the instantaneous force $[\mathrm{kgf} / \mathrm{s}]$ and the change in the knee and ankle angles for Less 100\% and Over 100\% was examined, but no statistically significant correlation was observed in any of the evaluation items. As mentioned above, this test also has additional data, especially in the vicinity of $1000[\mathrm{kgf} / \mathrm{s}]$, so we want to try to increase the number of participants in the experiment and increase the accuracy of feature extraction from the acquired data.

Here, the ratio of the pedaling force [\%] was $100 \%$ for the first time and $100 \%$ for the second time (Less $100 \%\left(1^{\text {st }}\right.$ time: $110 \%, 2^{\text {nd }}$ time: $\left.73 \%\right)$ ). Figure 5 shows the time history of the knee angle change (dotted line) with respect to the ankle joint angle change (solid line). The time when the lamp was turned on and the lower limb movement was started was set to 0 [s], and the first lower limb angle was set to $0\left[^{\circ}\right]$ so that the characteristics of the angle change from that time were easily extracted. As a result, in the first time of Over $100 \%$ compared to the second time of Less $100 \%$, the ratio of the change in the knee joint angle from 0 [s] to the change in the angle of the entire lower limb (knee + ankle joint) was large. In other words, it is considered that a strong tread force was generated by extending the knee joint.

Next, focusing on the instantaneous power $[\mathrm{kgf} / \mathrm{s}]\left(1^{\text {st }}\right.$ time: $1705.8 \mathrm{kgf} / \mathrm{s}, 2^{\text {nd }}$ time: $338.0 \mathrm{kgf} / \mathrm{s}$ ), when the same consideration is made, the first time is better than the second time. As in the case of the treading force, the ratio (contribution) of the change in the knee joint angle from 0 [s] to the change in the angle of the entire lower limb (knee + ankle joint) was large. From these facts, in order to apply the brake quickly, it is thought that it is better to bend the knee joint and then perform the braking operation to extend the knee joint firmly. A similar tendency can be seen in the time history data of other experiment participants. In addition to the percentage of treading force [\%] and instantaneous force $[\mathrm{kgf} / \mathrm{s}]$ discussed in this paper, the lower limb angle per hour. It is necessary to consider the rate of change in the evaluation as an evaluation index for the follow-up test.

\section{Conclusions}

An emergency braking operation experiment was performed by five test participants Lower limb movement [degree]

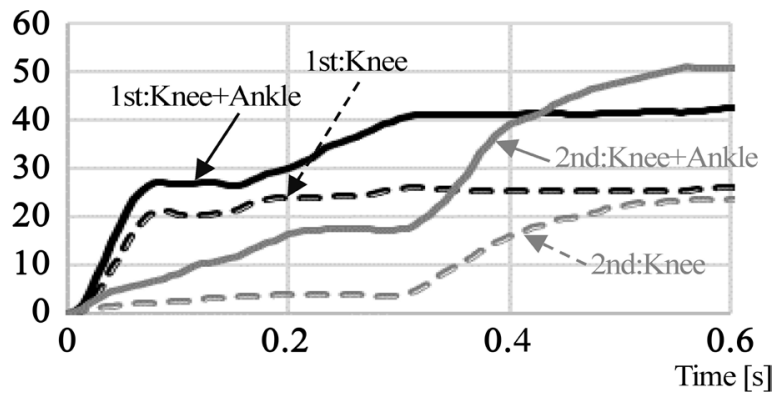

Figure 5. Changes in joint angles during brake operation. 
(young people). The results of statistical analysis of treading force and the maximum value of the change in the angle of the lower limb showed that the bending force was increased when the knee was bent, then the knee was extended, and the treading force was increased. Furthermore, it was shown that if the brakes were operated without moving the ankle joint, the operation would be quicker.

In addition, from the time axis data of each participant, it was shown that using the knee joints might be effective for performing the braking operation strongly and quickly.

In addition to increasing the number of participants in the experiment, additional measurements will be taken, such as changes in the angle between the trunk and thigh (hip joint), the relative position between the participant and the seat, and evaluation indexes based on time series. It was suggested that the possibility of clarifying the characteristics of the data that was unclear in this paper could be clarified by adding.

\section{Conflicts of Interest}

The authors declare no conflicts of interest regarding the publication of this paper.

\section{References}

[1] Cabinet Office (2017) White Paper on Aging Society 2017.

[2] National Institute of Social Security and Population (2016) Social Security Cost Statistics.

[3] Ministry of Health, Labor and Welfare (2016) Health and Welfare White Paper 2016.

[4] ITARDA INFORMATION (2016) Traffic Accident Analysis Report, Vol. 119.

[5] Traffic Accident Analysis Center (2017) Traffic Statistics.

[6] Yuichi, K., Shigeki, H. and Tsuyoshi, Y. (2017) Comparison of Impact Kinematics between Non-Obese and Obese Occupants in Frontal and Lateral. IRCOBI Conference, Antwerp, 13-15 September 2017, 91-103.

[7] Kent, R., Lee, S.H., Darvish, K., Wang, S., Poster, C., Lange, A., Brede, C., Lange, D. and Matsuoka, F. (2005) Structural and Material Changes in the Aging Thorax and Their Role in Crash Protection for Older Occupants. Stapp Car Crash Journal, 49, 231-249. https://doi.org/10.4271/2005-22-0011

[8] ITARDA INFORMATION (2018) Traffic Accident Analysis Report, Vol. 124.

[9] Wu, J.-R., Shinpei, K., Ya, Y.J. and Satoshi, T. (2011) Examination of Pedal Arrangement and Aging Effect of Car Braking Behavior in Emergency. Transactions of the Japan Society of Mechanical Engineers, 777, 442-450. https://doi.org/10.1299/kikaic.77.2062

[10] Zheng, Y., Kobayashi, T., Tamura, M. and Seto, Y. (2004) Study on Reflection Detection of Braking Operation. Transactions of the Society of Automotive Engineers of Japan, 35, 185-190.

[11] Nishikawa, K., Norasawa, T., Abe, H., Furukawa, K., Miyamoto, K. and Miyazaki, T. (2005) Study on Human Characteristics in Pedal Operation. Mazda Technical Re- 
port, No. 23.

[12] Hirakawa, A. (2017) Characteristics of, and Measures against Accelerator and Brake Pedal Misapplication Accidents. ITARDA 20th Work Shop Report, Tokyo, p. 1-15.

[13] Sekine, Y., Shibazaki, H., Ito, S. and Hirakawa, A. (2017) Analysis of the Influence of Elderly Sitting Posture on Pedaling Mistake. Proceedings of the 26th Annual Conference of the Japan Society of Mechanical Engineers, Transportation and Logistics Division, Tokyo, 12 April-12 June 2017, No. 17-53, p. 11. 